\title{
An Intelligent Information Management System for Retinal Image Storage and Recognition in Chronic Disease Using Digital Signal and Image Processing
}

\author{
Xiaojun Xu, Zhong Tang and Xijia He \\ Guangxi Medical University, Country China \\ E-mail: xuxiaojun51@gmail.com, ztang829@gmail.com, xijiahe5@gmail.com
}

Haixuan Wang

School of Management, Furen University, Xinbei, Taiwan 242304

E-mail: haixuanwang@outlook.com

Anil Sharma

Department of computer science, Faculty of Technology, Debre Tabor University, Ethiopia

E-mail: anilsharma@dtu.edu.et

Keywords: retinal imaging, retinal vasculature changes, chronic disease management, chronic disease information system, retinal blood vessels

Received: May 21, 2021

Retinal imaging plays a very significant role in the study of retinal vasculature changes indicative of chronic disease information related to vision. This study investigates the invulnerability of retinal image information in the disease information system and assesses the quantitative method of the morphological changes in the retinal vascular network. In this work, the medical digital image transmission protocol Digital Imaging \& Communications in Medicine (DICOM) version 3.0 and the retinal image Picture Archiving \& Communication System (PACS) were constructed in the laboratory using browser/server mode. Also, the DICOM-SR document was designed in this article using a list or hierarchy, and the retinal images to report the information of patients by using the Hypertext Transfer Protocol (HTTP) based Web Access to DICOM Persistent Objects (WADO) approach. The results showed that the retinal image PACS system constructed in Browser/Server mode can effectively store and transmit DICOM images. When the imaging device is combined with the application program, special adapters are used to negotiate the transmission syntax. The message flow is decoded in the communication process, which can be connected with the realization to improve the efficiency of information collection. The proposed PACS system integrates the quantitative features of retina providing more meaningful research data for data mining in comparison to the traditional state of the art methods based on chronic disease management system. The diagnostic ability of the retinal imaging procedure using the DICOM images is justified by obtaining 98.51\%, 98.04\%, 99\% and 99.01\% of accuracy, sensitivity, specificity and precision values respectively.

Povzetek: V prispevku je predstavljen protokol in sistem za shranjevanje slik očesnega ozadja za medicinske namene.

\section{Introduction}

Clinical retinal digital images are usually obtained by digital ophthalmoscopy, which is taken by a special camera through the pupil. At present, many ophthalmic image management systems mainly use fundus workstation equipment for collection and analysis, and most of the images are implemented in JPEG and other formats, which cannot be shared in hospitals, greatly limiting the analysis and utilization of retinal image data $[1,2]$. The conventional ophthalmic retinal imaging system illustrated in Figure 1 shows that the incident light from the retinal fundus possesses somewhat distorted wavefront which is further subjected to the adaptive mirror which is connected to the control system. Then the beam-splitter splits the waveform into two: one is sent to the waveform sensor and the other one is the corrected waveform obtained that results into a highresolution retinal image which can be stored for future usage $[3,4]$.

A basic schematic diagram of ophthalmic retinal imaging system is presented in Figure 1. 


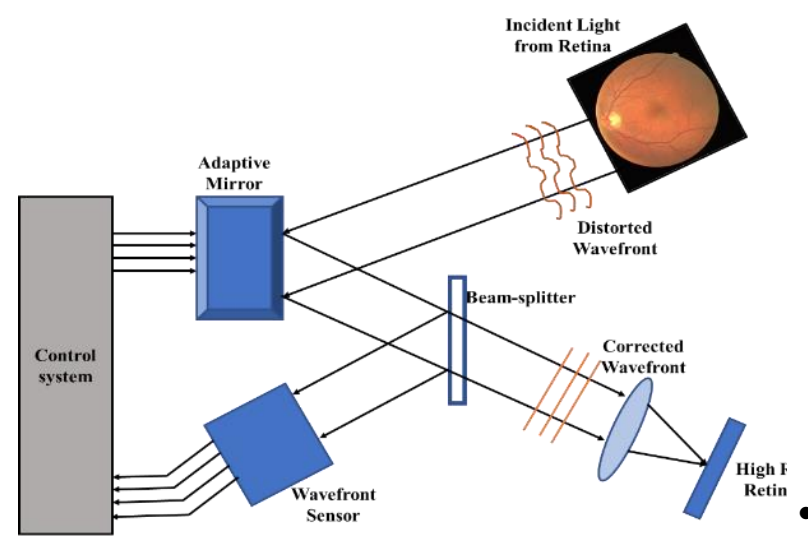

Figure 1: Schematic diagram of ophthalmic retinal imaging system.

The effectual retinal image management and storage is effective for the recognition of chronic diseases like Glaucoma, Diabetic retinopathy, macular degeneration and many more $[5,6]$. One of the greatest challenges in effective detection and diagnosis of various retinal diseases is their asymptomatic nature before it approaches the severe chronic disease levels [7,8]. However, different researches thrived for devising various diagnostic and image recognition systems for retinal image classification, management and storage. The fundus imaging-based methods are widely being used by the technological experts for retinal image screening [9]. A latest research in the field of classification of diabetic retinopathy severity of retinal imaging address the challenges of retinal landmark segmentation, retinal discrimination and severity grading while employing the hierarchal discrimination framework [10].

In order to solve the problem of registration of retinal images with different Angle of view of the retinal camera, Zou Beji et al. proposed a local feature registration method with affine invariability [11]. Then the anisotropic image structure is transformed into isotropic image structure with different angle factors by rotation, sampling and compression [12]. Finally, the scale-invariant feature transformation algorithm was used to extract feature points from isotropic image structures and match the feature point coordinates [13]. The experimental results in the private sample set containing 83 sets of retinal images showed that the root-meansquare error of this method was $1.247 \pm 0.251$ pixels, which could well solve the problem of perspective and scale change [14]. Liu et al. discussed the methods of noise reduction in the spatial and frequency domain of OCT images, and summarized the methods of precise localization and stratification of each layer of tissue in the OCT anterior segment and retina images [15]. In this paper, the hierarchical methods based on gray value search method, active contour model, graph theory and pattern recognition are deeply analyzed, and the advantages and disadvantages of existing hierarchical methods and existing problems are deeply discussed, and corresponding solutions and optimization schemes are proposed [16]. To analyze and evaluate the clinical diagnostic indicators of ophthalmology-related diseases, and further discuss and analyze the development trend and level of OCT image processing in the future according to the clinical demand of ophthalmology and the status quo of OCT image processing [17].

The motivation behind this article is to present the study based on medical information in the image transmission protocol (DICOM 3.0) and open source DICOM server which were not addressed in the previously presented state-of-the-art schemes. The major contributions of this work are:

- This work utilizes the eye-PACS architecture and uses the network Access based on HTTP DICOM Persistent objects (Web Access to DICOM Persistent Object WADO), which is connected [14] by developing a WADO network gateway interface.

- The main contribution of this study is retinal vascular analysis indicating chronic diseases, by investigating the invulnerability of retinal image information and assessment of quantitative method of the morphological change of retinal vascular network.

- The DICOM-SR document was designed using a list or hierarchy, and the retinal images and report information of patients were accessed using the Hypertext Transfer Protocol (HTTP) - based Web Access to DICOM Persistent Objects WADO approach.

- This work significantly contributed in the interoperation of retinal image information between heterogeneous chronic disease information systems, which were not addressed in the previously presented related work in this particular domain.

The rest of this article is organized as: Section 2 presents the related works in various domains of retinal imaging. Section 3 consists of material and methods comprising the architectures and flowcharts of designed modules. Results, analysis and discussion part is covered in section 4 followed by concluding remarks in section 5 .

\section{Related work}

Various state-of-the-art work in the field of retinal image Web-PACS, DICOM-SR designing, segmentation of retinal vasculatures, arteriovenous classification is presented in this section.

\subsection{Retinal image web-PACS and design of DICOM-SR}

Body circulation diseases often occur with retinal vascular changes, the retinal image as a vascular image can be obtained in vitro; the test in the diagnosis and treatment of diseases such as hypertension, diabetes evaluation is of great significance. In diabetic patients with retinal every once in a while, for six months or a year is about to do a fundus examination, hard physical labor took an ophthalmologist. Computer aided diagnosis is of great importance to solve this problem, including fundus retinal blood vessels is accurate and rapid automated segmentation technology. As early as in 1989, 
Chaudhuri et al. proposed a kind of retinal blood vessels detection method based on matched filter [18]. Wang and others are using multi-resolution Hemiite model to modeling and tag vessels [19]. Lam, and others use the gradient vector detect and extract the centerline vascular structures, further eliminate noise segmentation results using the centerline pruning technology. Boykov et al., in 2004 an interactive image segmentation method based on graph cut is proposed in [20].

The rapid development in artificial intelligencebased disease management and diagnosis assistant methods for standardizing and monitoring the real time health for immediate treatment of retinal disorders. Various feature-based image registration techniques like feature-based image registration utilizing global-local matching, model refinement using the hierarchal methods and dual- bootstrapping are particularly effective in the identification of sufficient number of vasculature features for disease discrimination. Various optimized image features were estimated for the registration of retinal fundus. Powell [21] derived the retinal vessel centre line distance for finding out the vasculature branching points as a landmark for image correspondence. The approach presented in [22] was tested for 462 pairs of the green channelled fundus images and reliable outcomes are achieved for the retinal image registration based on the identified vascular features for $2 \mathrm{D}$ vessel segmentation.

The related work section provides the detailing of various retinal image registration, management and storage approached for the recognition of chronic diseases like Glaucoma, Diabetic retinopathy, macular degeneration. The challenges posed by the state-of-theart methods reported in the literature lies in effective detection and diagnosis of various retinal diseases due to the asymptomatic nature of severe chronic disease levels. There are various researches being carried out in the field of retinal imaging for effectual analysis and timely treatment. To solve the problem of registration of retinal images with different Angle of view of the retinal camera, this work exploits the medical information in medical digital image transmission protocol (DICOM3.0). This experimentation adopts the model of browser/server $(\mathrm{B} / \mathrm{S})$ architecture of the retinal image PACS system in the laboratory. For retinal images of DICOM format, use DICOM gateway after processing integrated into PACS. Also, this experiment is to the design of DICOM SR document using a list or hierarchy, the basic unit is the "item" (the content item). Each item includes name/value and relationship using WADO manner based on HTTP connections, through the development of network gateway with WADO interface. WADO requests will be made through the HTTP GET method implementation and the users in the image list page by clicking on the link. Then send WADO requests to the server and the server returned response directly on the page. When the user wants to enter the patient list JSP view, they just ask the DICOM archive server sends the request form, which provides access to the patients with retinal image and report information.

\subsection{Retinal vascular segmentation and arteriovenous classification}

Retinal blood vessels segmentation is the basis of accurate quantitative analysis of retinal vascular. Uneven illumination in retinal fundus photography network of blood vessels by arteriovenous GuanShu cross makes the characteristics of automatic segmentation of retinal arteriovenous became very difficult. According to the above characteristics, in this topic, we obtain the retinal image of uneven background correction, image green channel extraction, and the image on the retina of discrete wavelet transform to enhance the sampling. By extracting pixel gray value, retinal vascular neighborhood two-dimensional gray entropy texture feature, and by using fuzzy c-means clustering algorithm for clustering, extract retinal blood vessels. The framework first use morphological image processing on the optic disc structure on the retinal image automatic segmentation and extraction. Then, different labeled seed points were used as the initial points to track the segmented vessels of different types, and the retinal vascular network was re-labeled to provide a basis for the subsequent extraction and analysis of retinal arteriovenous morphological features [21].

\subsection{Quantitative analysis of retinal vascular tortuosity}

In clinical ophthalmology work, usually the first change of vascular patterns is vascular contorts change. Eye doctors commonly used qualitative index to describe the change, such as mild circuitry, moderate, severe and extreme circuitry gives an index of the severity of diabetic retinopathy. To give the characterization, the curvature is the main mathematical models of retinal blood vessels. This study will retinal vascular centerline as a random process, first of all, according to the image neighborhood information to extract the blood center line and then the vascular segment between two adjacent feature points is automatically separated to extract the pixel of each pixel on the Central Line of the segment coordinate information, and by using the second order moments of the random process metrics such as the variation of statistical features to reflect the degree of change curve, through the simulation curve data and actual test on the retinal image, the test after the experiment methods for different scale, rotation of vascular give stability, and compared with other indices like chord is reported in the literature [22].

\subsection{Quantitative analysis of retinal vessel width}

Retinal vessel width and Artery-to-Vein diameter Ratio (AVR) for the diagnosis of chronic diseases, especially the change of blood pressure diseases such as hypertension is of great significance. The retinal images can be directly through the noninvasive fundus examination, thus convenient vascular morphology of them is analyzed. This paper measures the blood vessels cross section of the pixel gray value distribution curve 
can appear due to retinal artery reflective phenomenon, so this experiment using the Gaussian mixture model of grey value distribution curve fitting. First, the vascular tree is utilized according to the bifurcation point is broken, and then from the patrol vessels branches automatic calculation area and main shaft length, then further obtain vascular branches. The average width of the value we will compare different width, the consistency of the measurement results.

\subsection{Quantitative analysis of retinal blood vessels}

Retinal image is a kind of biological image, has certain anatomical characteristics of retinal vein embarks from the optic disc, gradually outward radiation, and the lower branches, blood vessel diameter decreases, the tree structure, the small blood vessels in general is a branch of the great vessels that branch of the tree structure is rich, the entire network of blood vessels in complex structures, and topology variety, not easy words to describe. In clinical work, general ophthalmic clinicians with macular and optic disc regional center as the center, to observe the retinal blood vessel image of the up and down or so four quadrant vascular lesions of the abnormal lesions. At present, many different studies have shown that no retinal image lesions with area have different diagnostic significance. In addition, fractal dimension of retinal vascular network is analyzed to know the changes occurring due to chronic diseases [15].

\section{Materials and methods}

This section includes the architecture of various modules like; Web-PACS for Retinal Images based on browser/server mode, designing of DICOM-SR for Retinal Vascular Images.

\subsection{Architecture of Web-PACS for retinal images based on $\mathrm{B} / \mathrm{S}$}

In this experiment, the Browser/Server $(\mathrm{B} / \mathrm{S})$ mode was adopted to construct the retinal image PACS system, which mainly included the client layer, the Server layer and the data layer presented in Figure 2.

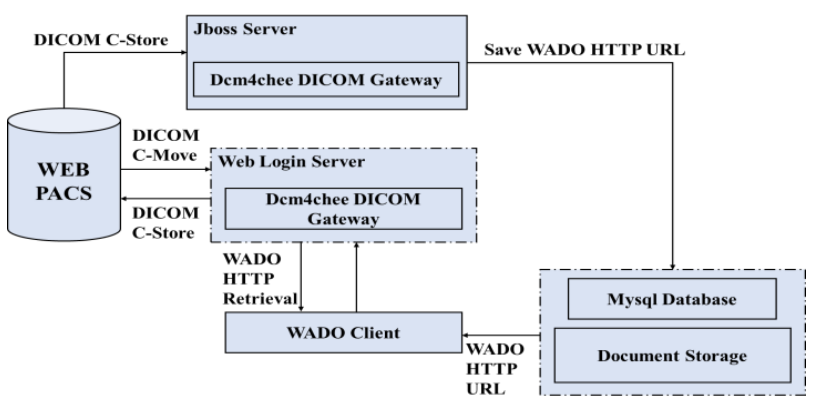

Figure 2: System Design Diagram for Web-Pals.

The client layer mainly includes a variety of $\mathrm{Web}$ browser software to support requests to access the middle tier services. In this experiment, Microsoft's Internet explorer browser, 360 browser and Google Chrome browser were installed on the desktop computer in the laboratory, safari was installed on the ipad tablet computer, and UC browser was installed on the android operating system mobile phone. For the server layer, the data layer is primarily connected through the Open Data Base Connectivity (ODBC) protocol and is serviced by the client layer over the HTTP/HTTPs protocol. In this experiment, we used dcm4chee open-source server software to provide WADO access resolution service for network customers, processed service requests from the customer layer and made corresponding responses, and provided various information needed to access customers through the designed interface.

In this experiment, a Lenovo TS230 (Windows7 operating system, Intel Core i3 processor, 2GB of memory, and 50OGB of hard disk) was installed as the server in the laboratory, and the J2EE enterprise version servlet engine support JSP running environment was installed on it. Web cache and JBOSS server program were also installed to support WADO application in the Web environment. The data layer includes DICOM storage to store DICOM inspection, image series and other data. The database and services for this experiment are installed on a workstation, and the database management system used is an open-source MySQL program. The conversion of retinal JPEG images to DICOM images is realized by using DICOM gateway and transmitted to PACS.

\subsection{Design of DICOM-SR for retinal vascular images}

Fundus image diagnosis report contains a large amount of medical information reflecting the fundus characteristics of patients, but the current image diagnosis report USES the narrative natural language or text data of medical personnel, making it difficult for the computer to extract, analyze study and classify them. With the popularity of PACS system and the continuous upgrade of DICOM standard, DICOM3.0 standard also formulated an image diagnosis result management service class for the management of diagnostic report. In 2000, Information Object Definitions (IODs) of Structured Report (SR) was added to DICOM3.0 standard, providing a basis for computer to understand and express image diagnostic reports as presented in Figure 3.

Another important purpose of the design of retinal image DICOM-SR is to provide a basis for Computer aided diagnosis (CAD). In the diagnosis of retinal images, it is of great value to provide reasonable templates for clinicians. In this experiment, the CAD-SR template for eye foundation disease was designed according to the tree structure. Starting from the root of the file, it was divided into four parent nodes, and then each parent node had several child nodes presented in Figure 4. 


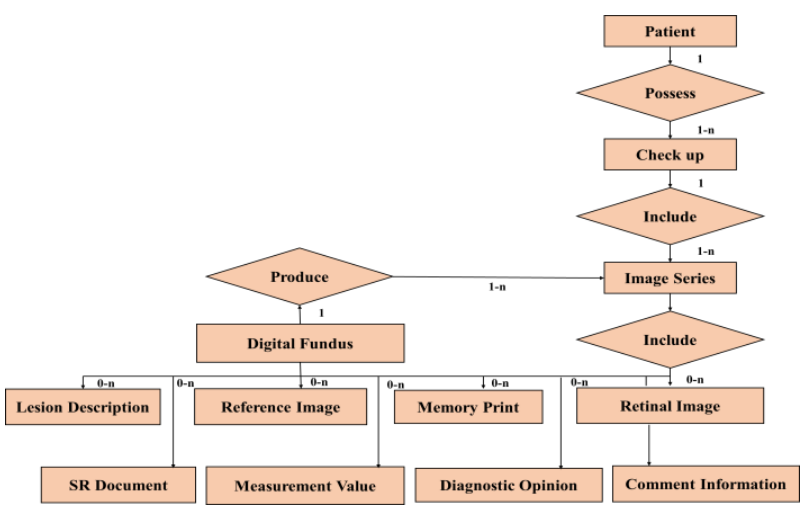

Figure 3: e-r model of retinal DICOM-SR.

In terms of function, the process designed in this experiment (Figure 3-5) is the blood vessel quantitative analysis module provided by the system after the system receives the image, which can be used for quantitative analysis of retinal blood vessels. For complex fundus lesions, doctors can detect and extract the lesions with the help of retinal image processing algorithm provided by the system, and measure the lesions.

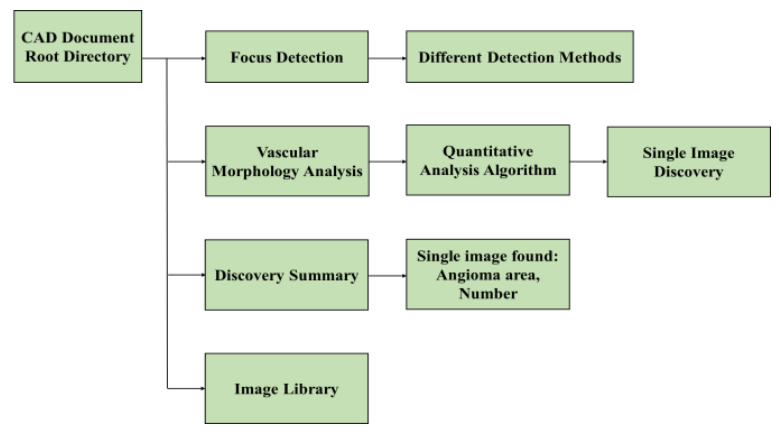

Figure 4: Design framework of retinal image CAD-SR document.

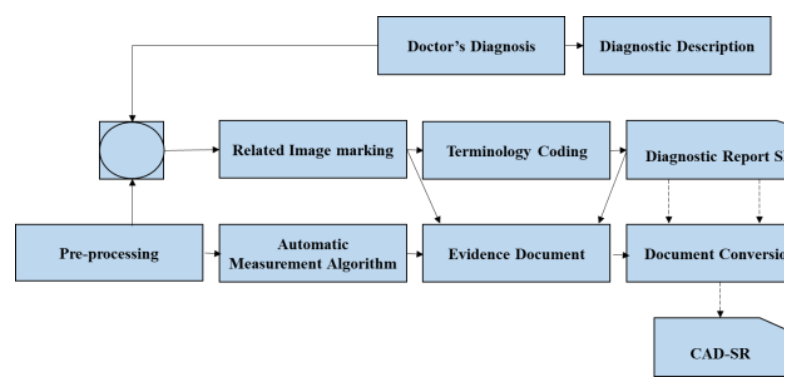

Figure 5: DICOM model business process diagram of CAD-SR.

Figure 5 depicts the DICOM model business process diagram of CAD-SR. In addition, doctors can also use the fundus lesions and fundus structure for text description. The above text and numerical information can be stored into PACS data in DICOM-SR format for easy query and transmission. For retinal image report files, graphic reports are usually required. To this end, this experiment designed a structured report document model with report content and image. The text information in the file is populated into the XSLT style sheet. The experimental design of retinal image type is contained in the SR, as a result, the XSLT part of the image is generated from the WADO image requests. It is seen in the form of XML to the browser side, in order to access the users to browse information.

\subsection{Fundus image prepossessing}

\section{A. Normalization of image color}

Because images of retinal blood vessels produced by different imaging devices can be affected by light and shadow, this will affect the image feature extraction algorithm based on seeds point resulting in heavy play effect. Therefore, by the experiment of the retinal image of the need to be normalized RGB color space. The retinal image depicting different $\mathrm{R}, \mathrm{G}$, and $\mathrm{B}$ channels after image color normalization are shown in Figure 6 along with their respective histograms.

B. Normalization of image scale

Due to different imaging equipment of retinal blood vessel image resolution may be different, on the back of the feature extraction algorithm based on seeds point based on different image width results have important influence. Therefore, need to experiment by the scale of the retinal image space normalized processing, the experiment of all processing image pixel is adjusted for unified $539 \times 539$ pixel size.
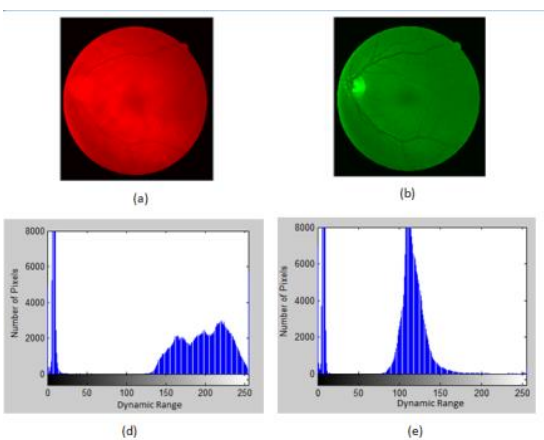

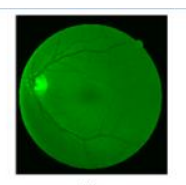

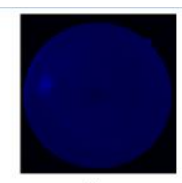

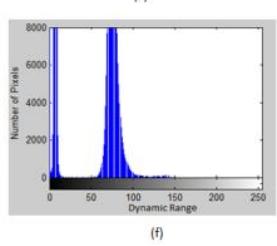

Figure 6: Image color normalization into R, G, B channels (a) Red channel (b) Green Channel (c) Blue Channel (d) Histogram of Red channel (e) Histogram of Green channel (f) Histogram of Blue channel.

\subsection{Extraction of optic disc in retinal images}

Optic disc (OD) is located at the oracular area nasal side about $3 \mathrm{~mm}$ and $1.5 \mathrm{~mm}$ in diameter, is the start of a central retinal blood vessels into the fundus. Under normal circumstances, $0 d$ on the fundus image boundary clear, white, disc. This experiment by use of the morphological characteristics of $0 d$ first to $0 d$, which can identify the extraction of intravenous seed point lays the foundation for the next step. This method could leak will retinal image threshold segmentation of seepage clearly separated, the experiment of image processing, when the threshold $T$ is equal to 150 , has better segmentation effect, the result of the finalization image 
recycling used earlier in this paper. The mathematical shape of the image processing method to obtain the final OD segmentation results. The complete process of OD center identification, Boundary localization and OD segmentation is depicted in Figure 7.

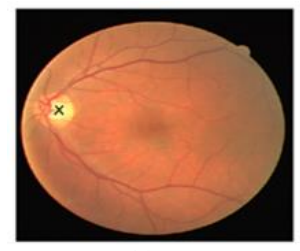

(a)

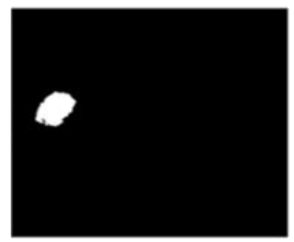

(c)

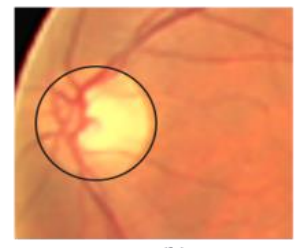

(b)

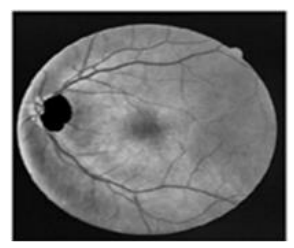

(d)
Figure 7: (a) Original Retinal image with located OD centre, (b) Cropped retinal area with OD boundary identified (c) OD segmented portion (d) Retinal image after OD segmentation.

\subsection{Retinal image report transmission based on WADO protocol}

WADO standard is a technical standard released by international DICOM standard organization in 2004, which is fully known as Web Access to DICOM Persistent Objects. As the 18th part of DICOM3.0 standard, WADO standard mainly defines a mechanism for storing and displaying DICOM Objects from HTML or XML documents based on Web through HTTP or HTTPS protocol. In addition to single frame images, the server can also respond to client requests with different types, sending multiple frames of images and text documents. Therefore, this experiment carried out relevant experiments of retinal DICOM image and structured report through WADO standard. Our framework based on IFIE ophthalmic technology (EYECARE TF, version 3.7) to confirm the function modules of the medical information enterprises subset, and made clear a series of mutual coordination between the medical enterprises, based on the standard of the transaction. IHE defines the DICOM queue from the service users (Supreme Court of the United States) and services (sarcoplasmic calcium-binding proteins) matching and comes back key, eligible keys used to select the query service instance of a class (instance) to users of the service industry, and then the key value returned back only specific data is not used for matching. Figure 8 illustrates the flowchart of retinal image/report under IHE specification.

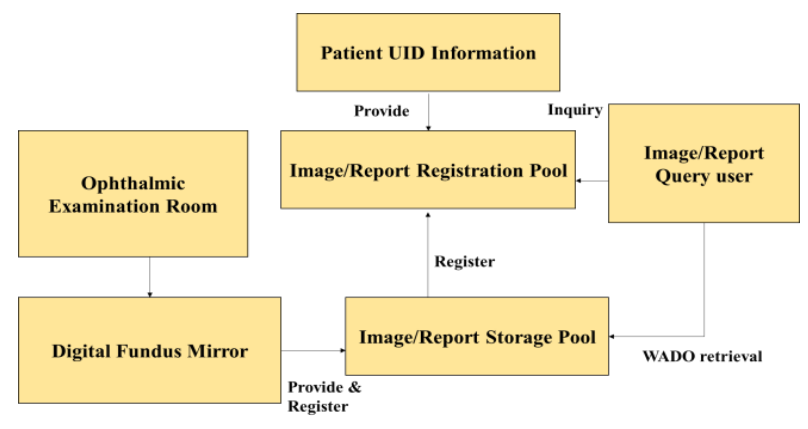

Figure 8: Flow chart of retinal image/report under IHE specification.

When the patient comes for fundus examination, the type of examination is first set, and the user queries the department or operation arrangement through the DICOM modal work list. The work list is downloaded into the imaging mode, and the user confirms the information. When a shoot transaction is executed, this information is written to the header section of the resulting object. Get the modal or get the modal import operation sends the retrieved modal Image to the Image Archive (< Image Archive) and appears in the header file information that produces the Image. After receiving the image display demand of retrieval operation, the DICOM image and measurement required are transferred from the image file to the image display module for display. Results and Analysis

This section illustrates the analysis of results obtained for retinal image Web-PACS, access and display of retinal image on DICOM-SR and finally presents its discussion and summary.

\subsection{Retinal image Web-PACS}

The functions of the retinal image PACS system in this experiment include: first, the function of storage and browsing. Open the PACS system and you can enter the work list of fundus images. After selecting the desired image catalog, you can display the pictures and reports of patients on the Web page. In addition, third-party DICOM browser can also be used to look up the patient picture by name, check mode and other means. The patient image is stored in the same way as the SR report and can be read at the same time. Second, the basic processing functions of retinal image realizes browserbased image processing functions, including window width and window level adjustment, manual measurement, zoom size, rotation and translation, negative display and other basic image processing functions. Third function is the communication which can realize remote communication function, image compression and transmission, download patient information and image retrieval and query function. In this study, the website is logged on through computer system with different operating system which can provide the fundus images and related reports conveniently. The Web-PACS system connected in the laboratory for the experimentation purpose is shown in Figure 9. 


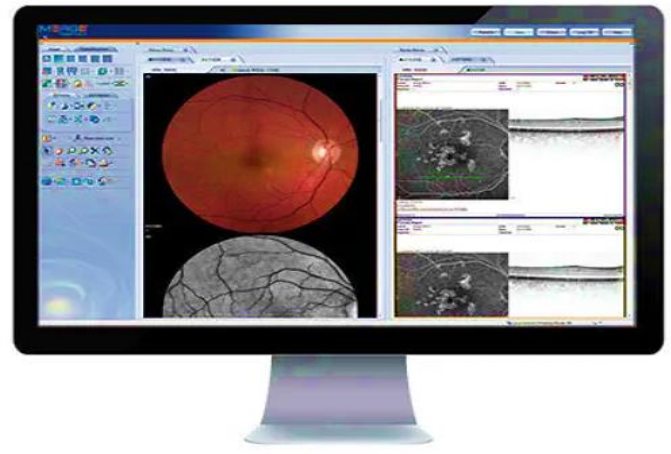

Figure 9: Web-PACS system connected in the laboratory.

\subsection{Access and display of retinal image DICOM-SR}

Retinal image transmission based on WADO standard can better solve the problem of heterogeneous medical information system accessing retinal image on the Internet. Based on the traditional PACS system, this standard defines how the Web client obtains the format and method of retinal image URL from the Web enabled DICOM server. In this experiment, retinal image DICOM browser and WADO Application server are both Application entities (AE) conforming to DICOM standard, which can realize the interaction function of heterogeneous medical information integration platform through the standard DICOM communication service. The basic structure of WADO is http://< server IP address $><$ path $>$ ? < query parameter $>$, where the study UID parameter is the UID of the object's check, and the series UID is the UID of the object's series.

For comparing the diagnostic ability of the retinal imaging procedure using the DICOM images, various evaluation parameters are taken into consideration like Accuracy, Sensitivity, Specificity and Precision. These values are derived from true positives (TP), true negatives (TN), false positives (FP) and false negatives (FN) values. The outcomes of the diagnostic ability in terms of recognition performance are depicted in Table 1.

\begin{tabular}{|l|l|}
\hline Evaluation Parameters & Recognition Value \\
\hline Accuracy & $98.51 \%$ \\
\hline Sensitivity & $98.04 \%$ \\
\hline Specificity & $99 \%$ \\
\hline Precision & $99.01 \%$ \\
\hline
\end{tabular}

Table 1: Outcomes of diagnostic ability in terms of recognition performance.

It is seen from Table 1 that the accuracy value of $98.51 \%$ is obtained for the retinal image recognition along with the sensitivity of $98.04 \%$, Specificity of $99 \%$ and precision value of $99.01 \%$. The viability of the accurate recognition rate and other parameters is justified by the outcomes obtained.

\subsection{Discussion and summary}

Different from the general medical imaging equipment, which directly integrates the images into the hospital PACS system, the object of this experiment is retinal images, which are usually produced in the ophthalmology and endocrinology departments of the hospital and generally stored on the workstation of the inspection equipment. Arvin et al. converted pathological images into DICOM images and uploaded them to the PACS system, realizing the information system integration of pathological images. Similarly, retinal image is integrated into PACS system by dicomization of retinal image. In addition, it is a better solution to use the image device with DICOM standard digital interface for direct transmission. The information exchange between application entities follows the Client/Server (C/S) pattern, with Service Class Consumer (SCU) and Service Class Provider (SCP) as Client Server roles. When the imaging device is combined with the application program, special adapters are used to negotiate the transmission syntax and how the message flow is decoded in the communication process, which can be connected with the realization to improve the efficiency of information collection.

In this article, we discussed the slow disease information management in the retinal image information and its significance, and then puts forward a framework of retinal image transmission based on WADO standards, and through the architecture of retinal image PACS system, realized the browser to access the retinal image and structured report, for retinal image information in a heterogeneous invulnerability laid a solid foundation in the health care system. The WADO standard adopted in this experiment is based on the Web and can store and display DICOM objects from HTML or XML documents through HTTP or HTTPS protocols. DICOM server set DICOM parser, can achieve DICOM to JPEG format conversion, so that the browser client can access the retina DICOM image. In order to enable browsers to access DICOM data through the WADO standard, WADO server components need to be designed and deployed so that users can access retinal images and report data using the Web on top of the existing information system. In this experiment, through the architecture of retinal image WADO application server conforming to DICOM standard, the interactive function of retinal DICOM image communication service based on Web was realized. In addition to the parameters used in the URL in this experiment, the URL can also be used to define the transfer syntax of the image (lossless/lossless compression), to define the luminosity and contrast of the image, the portion of the image that will be displayed, and to add annotations to the image.

\section{Conclusions}

The proposed WADO based retinal image transmission technology and structured numerical report in DICOMSR can better solve the invulnerability problem of retinal image in different systems. The analysis done in this 
work for the investigation of invulnerable retinal imaging information can be used for quantitative analysis of morphological change of retinal vascular network. This work is mainly focused on the medical digital image transmission protocol Digital Imaging and Communications in Medicine (DICOM) version 3.0 and the retinal image Picture Archiving and Communication System (PACS) was constructed in the laboratory. The retinal image PACS system constructed in B/S mode can effectively store and transmit DICOM images when combined with the application program. This project will integrate quantitative features of retina in future research, providing more meaningful research data for data mining based on chronic disease management system. In addition, a study will be conducted on the conversion of retinal images and reports based on DICOM 3.0 standard and HL7 CDA documents. Therefore, in order to provide a technical basis for the integration of retinal images and existing resident health records with HL7 interfaces. The feasibility of the recognition rate and other evaluation parameters is justified by obtaining the $98.51 \%$ accuracy rate with comparatively better values of sensitivity, specificity and precision.

\section{References}

[1] Tu, Y., Huang, Y., \& Yi, F. (2015, April). Chronic disease management system design based on cloud storage architecture. In 2015 2nd International Conference on Information Science and Control Engineering, pp. 654-658. IEEE. https://doi.org/10.1109/ICISCE.2015.151.

[2] Sharma, A., Ansari, M. D., \& Kumar, R. (2017, September). A comparative study of edge detectors in digital image processing. In 2017 4th International Conference on Signal Processing, Computing and Control (ISPCC), pp. 246-250. https://doi.org/10.1109/ISPCC.2017.8269683.

[3] Bhardwaj, C., Jain, S., \& Sood, M. (2020). Retinal blood vessel localization to expedite PDR diagnosis. Periodicals of Engineering and Natural Sciences (PEN), 8(3), 1233-1246. http://dx.doi.org/10.21533/pen.v8i3.208.

[4] Bhardwaj, C., Jain, S., \& Sood, M. (2019). Automatic blood vessel extraction of fundus images employing fuzzy approach. Indonesian Journal of Electrical Engineering and Informatics (IJEEI), 7(4), 757-771. https://doi.org/10.11591/ijeei.v7i4.991.

[5] Shah, A. R., \& Gardner, T. W. (2017). Diabetic retinopathy: research to clinical practice. Clinical diabetes and endocrinology, 3(1), 1-7. https://doi.org/10.1186/s40842-017-0047-y.

[6] Dogra, J., Jain, S., Sharma, A., Kumar, R., \& Sood, M. (2020). Brain tumor detection from MR images employing fuzzy graph cut technique. Recent Advances in Computer Science and Communications (Formerly: Recent Patents on Computer Science), 13(3), 362-369. https://doi.org/10.2174/22132759126661812071526 33.
[7] Bhardwaj, C., Jain, S., \& Sood, M. (2020). Diabetic Retinopathy Lesion Discriminative Diagnostic System for Retinal Fundus Images. Advanced Biomedical Engineering, 9, 71-82. https://doi.org/10.14326/abe.9.71.

[8] Bhardwaj, C., Jain, S., \& Sood, M.(2020). Automated Diagnostic Hybrid Lesion Detection System for Diabetic Retinopathy Abnormalities. International Journal of Sensors Wireless Communications and Control, 10(4), 494507.

https://doi.org/10.2174/22103279096661911260924 11.

[9] Sharma, A., Tomar, R., Chilamkurti, N., \& Kim, B. G. (2020). Blockchain based smart contracts for internet of medical things in ehealthcare. Electronics, 9(10), 1609. https://doi.org/10.3390/electronics9101609.

[10] Bhardwaj, C., Jain, S., \& Sood, M. (2020). Hierarchical severity grade classification of nonproliferative diabetic retinopathy. Journal of Ambient Intelligence and Humanized Computing, 122. https://doi.org/ 10.1007/s12652-020-02426-9.

[11] Mishra, A. R., Garg, A. K., Purwar, H., Rana, P., Liao, H., \& Mardani, A. (2021). An extended intuitionistic fuzzy multi-attributive border approximation area comparison approach for smartphone selection using discrimination measures. Informatica, 32(1), 119-143, DOI: https://doi.org/ 10.15388/20-INFOR430.

[12] Chung, K., \& Park, R. C. (2019). Cloud based uhealthcare network with QoS guarantee for mobile health service. Cluster Computing, 22(1), 20012015. https://doi.org/10.1007/s10586-017-1120-0.

[13]Zhang, S., Tang, J., Meng, F., \& Yuan, R. (2021). A group decision making method with interval-valued intuitionistic fuzzy preference relations and its application in the selection of cloud computing vendors for SMEs. Informatica, 32(1), 163-193, DOI: https://doi.org/ 10.15388/20-INFOR416

[14] Xiaosong, L., Gang, S., \& Yong, C. (2015, November). Design and development of the webbased health and chronic disease assessment management system. In 2015 7th International Conference on Information Technology in Medicine and Education (ITME), pp. 72-75. IEEE. https://doi.org/10.1109/ITME.2015.167.

[15]Liu, X., Zeng, H., Chand, N., \& Effenberger, F. (2015). Efficient mobile fronthaul via DSP-based channel aggregation. Journal of Lightwave Technology, 34(6), 1556-1564. https://doi.org/10.1109/JLT.2015.2508451.

[16] Gusyev, M. A., Morgenstern, U., Nishihara, T., Hayashi, T., Akata, N., Ichiyanagi, K., ... \& Stewart, M. K. (2019). Evaluating anthropogenic and environmental tritium effects using precipitation and Hokkaido snowpack at selected coastal locations in Asia. Science of The Total Environment, 659, 13071321. 
https://doi.org/10.1016/j.scitotenv.2018.12.342.

[17] International Society for Optics and Photonics. https://doi.org/10.1117/12.843714. Kalibatienè, D., \& Miliauskaite, J. (2021). A Hybrid Systematic Review Approach on Complexity Issues in DataDriven Fuzzy Inference Systems Development. Informatica, 32(1), 85-118, DOI: https://doi.org/ 10.15388/21-INFOR444.

[18] Koutelakis, G. V., \& Lymberopoulos, D. K. (2008). WADA service: an extension of DICOM WADO service. IEEE Transactions on Information Technology in Biomedicine, 13(1), 121-130. https://doi.org/10.1109/TITB.2008.2007197.

[19]Al-Rawi, M., \& Karajeh, H. (2007). Genetic algorithm matched filter optimization for automated detection of blood vessels from digital retinal images. Computer methods and programs in biomedicine, 87(3), 248-253.

https://doi.org/10.1016/j.cmpb.2007.05.012.

[20] Xu, Xin, Li Li, and Ashutosh Sharma. "Controlling messy errors in virtual reconstruction of random sports image capture points for complex systems." International journal of system assurance engineering and management (2021): 1-8. https://doi.org/10.1007/s13198-021-01094-y

[21] Powell, M. J. (1964). An efficient method for finding the minimum of a function of several variables without calculating derivatives. The computer journal, 7(2), 155-162. https://doi.org/10.1093/comjnl/7.2.155.

[22] Boncea, R., PETRE, I., SMADA, D. M., \& ZAMFIROIU ANAGRAMA, A. (2017). A Maturity Analysis of Big Data Technologies. Informatica Economica, 21(1),DOI:

https://doi.org/10.12948/issn14531305/21.1.2017.05 
